\section{The inosine conundrum}

\section{By Kai-Jye Lou, Staff Writer}

Inosine has been known to promote recovery in rat ischemic stroke models, in which broad swaths of the brain are affected, but it remained unclear whether the compound might restore functions mediated by specific brain regions. Researchers at the Children's Hospital Boston and the University of California, Los Angeles have now used a more refined stroke model to show that inosine can restore functions that are localized to precise areas in the brain. ${ }^{1}$

The new findings provide additional rationale for investigating inosine's effects in humans, but the path forward remains uncertain. Alseres Pharmaceuticals Inc. has been developing inosine applications since 2000, but it is cash strapped and is seeking a partner. Meanwhile, some researchers think it might be better to start from scratch and develop an inosine derivative that has a longer half-life.

The purine nucleoside activates serine/ threonine kinase 24 (STK24; MST3B), which promotes the growth of axons to denervated brain stem and spinal cord regions in vivo., ${ }^{2,3}$

An earlier study from a research group led by Larry Benowitz, who is also senior author on the current paper, showed that inosine improved performance in several tasks designed to assess recovery in the post-stroke setting, including reaching ability and limb placement. ${ }^{2}$

However, the rats used in that 2002 study had widespread damage that spanned multiple major brain regions responsible for controlling a broad range of body functions. The widespread damage confounded the researchers' ability to assess whether the molecule could speed the recovery of a function that was controlled by a specific strokedamaged region.

"What we knew before was that if we create a massive stroke on one side of the brain, inosine would promote axon growth from the undamaged side to the damaged side and promote some degree of recovery," said Benowitz, director of the laboratories for neuroscience research in neurosurgery at Children's Hospital Boston and an associate professor of neurosurgery at Harvard Medical School. "But what we didn't know was whether inosine would allow for the recovery" of functions previously controlled by a specific region of damaged brain.

To address this question, another Benowitz-led team used a photothrombotic stroke model to induce a more localized, unilateral ischemic lesion centered on the forelimb motor area in the rat brain. The animals were then evaluated for their ability to retrieve food pellets - a task that requires a functional forelimb motor area.

An intraventricular bolus injection of inosine post-stroke followed by constant infusion using osmotic minipumps restored the food pellet retrieval ability in rats with the stroke-impaired forelimb to about $80 \%$ of baseline compared with $35 \%-40 \%$ of baseline in saline-treated controls $(p<0.001$ by week 4$)$. The effects persisted for at least a month.

The findings were published in The Journal of Neuroscience.

\section{Different window, different endpoints}

Most therapeutics that have been put into development for acute ischemic stroke have been clot busters or neuroprotectants designed to limit the extent of damage. But, in part because of the limited treatment window, only one drug-Activase alteplase from the Genentech Inc. unit of Roche-has made it to market so far. The recombinant form of human tissue plasminogen activator (tPA) is indicated for use only within three hours of stroke.

In contrast, inosine targets the neural repair pathway. "That's the exciting thing about inosine-the temporal window for neural repair is wider. A question to answer, though, is how wide the clinical window is for treatment with inosine to be effective," said S. Thomas Carmichael, an associate professor of neurology at UCLA.

Answering that question has been problematic, however, as inosine hasn't had a smooth path in the clinic.

Children's Hospital Boston and Alseres separately hold multiple issued and pending patents covering inosine and its use in traumatic CNS injuries. And the company holds exclusive worldwide rights from the hospital for the use of inosine in stimulating nerve repair.

Following a 2004 IND submission from Alseres (then Boston Life Sciences Inc.), the FDA placed the program on hold pending the submission of additional preclinical pharmacology and toxicology data. The clinical program had intended to evaluate inosine delivered via an implantable subcutaneous pump and intracerebroventricular catheter system.

Alseres submitted additional data to the FDA in 2005, but the agency wanted more preclinical data. The company did not submit a subsequent data package, and the clinical hold on the original IND has not been removed.

Instead, Alseres has switched to a high-concentration formulation of inosine that is intravenously administered. The program is now in preclinical development for traumatic CNS injuries like spinal cord injury, traumatic brain injury and stroke.

Despite the setbacks, Armin Schneider, VP of molecular neurology at Sygnis Pharma AG, thinks molecules that improve 


\section{TARGETS \& MECHANISMS}

post-stroke rehabilitation are worth pursuing. "Patients receive physical or speech-based therapy in a rehabilitation setting," he said. "So far, no pharmacological enhancement of recovery processes is available, and any new available method for enhancement of recovery would be a highly valuable addition."

However, Schneider noted that the design of clinical trials for such compounds is relatively uncharted territory.

"In the acute stroke setting, it is very clear what the FDA and other regulatory agencies want to see-significant improvements in the modified Rankin Scale and the National Institutes of Health Stroke Scale," he said. "However, there is not much data in the literature that tells you how to design clinical trials for post-stroke rehabilitation. It is unclear what would be considered a medically relevant benefit to the patients. This is a novel field and the endpoints are difficult to define."

Sygnis' AX200 is in Phase II testing for acute ischemic stroke. The G-CSF also has completed an investigator-led exploratory study in humans for stroke rehabilitation, and the data from this study are currently being analyzed.

\section{Delivery concerns}

In addition to trial design challenges, questions remain about how best to deliver inosine.

Alseres did present data in 2008 showing that rats receiving i.v. delivery of a high-concentration formulation of inosine had over $600 \%$ increases in levels of the molecule in the brain. ${ }^{4}$ Whether this increase is enough for a therapeutic effect remains to be demonstrated.

Benowitz, who was a member of Alseres' scientific advisory board until last March, said his group is evaluating the company's high-concentration inosine formulation in in vivo animal models.

In any case, Schneider suggested that systemic delivery routes are likely to be challenging because of the small molecule's short plasma half-life. "Greater than $95 \%$ of inosine is cleared from the plasma within 5 minutes," he noted. "I don't think systemic delivery for inosine will be possible, as the compound is metabolized so fast that you likely won't be able to get sufficient amounts of it into the brain."

Thus, Schneider thinks it may be better to address the delivery and formulation issues by starting from scratch. "It may be possible to develop an analog that targets MST3B, that crosses the blood brain barrier and does not undergo the same metabolic processes as inosine," he told SciBX.

Regardless of how inosine is given, Schneider said the timing of therapy will need to be worked out.

"The current study initiates treatment 30 minutes after onset of ischemia," he noted. "Additional studies are needed in animals subjected to cerebral ischemia where treatment is initiated at delayed time points after ischemia, like days and weeks. It might also be interesting to see if there are effects on learning and memory performance after stroke."

Resolving those issues in the clinic apparently will have to wait until Alseres finds a partner with the wherewithal to take inosine forward. The company had about $\$ 1$ million in cash on May 12 and has since raised an additional \$800,000. For the period ending March 30, Alseres reported a three-month operating loss of $\$ 4.3$ million.

Lou, K.-J. SciBX 2(29); doi:10.1038/scibx.2009.1132

Published online July 30, 2009

\section{REFERENCES}

1. Zai, L. et al. J. Neurosci.; published online June 24, 2009; doi:10.1523/JNEUROSCI.0414-09.2009

Contact: Larry Benowitz, Children's Hospital Boston, Boston, Mass. e-mail: larry.benowitz@childrens.harvard.edu

2. Chen, P. et al. Proc. Natl. Acad. Sci. USA 99, 9031-9036 (2002)

3. Smith, J.M. et al. Brain 130, 915-925 (2007)

4. Cusack, N. et al. Neurotherapeutics 5, 494 (2008)

\section{COMPANIES AND INSTITUTIONS MENTIONED}

Alseres Pharmaceuticals Inc. (Pink:ALSE), Hopkinton, Mass.

Children's Hospital Boston, Boston, Mass.

Food and Drug Administration, Silver Spring, Md.

Genentech Inc., South San Francisco, Calif.

Harvard Medical School, Boston, Mass.

Roche (SIX:ROG; OTCQX:RHHBY), Basel, Switzerland

Sygnis Pharma AG (Xetra:LIO), Heidelberg, Germany University of California, Los Angeles, Calif. 